\title{
Role of a triangle singularity in the $\pi N(1535)$ contribution to $\gamma p \rightarrow p \pi^{0} \eta$
}

\section{S. Sakai*, V.R. Debastiani, and E. Oset}

Departamento de Física Teórica and IFIC, Centro Mixto Universidad de Valencia-CSIC

Institutos de Investigación de Paterna, Apartado 22085, 46071 Valencia, Spain

E-mail: shuntaro.sakaidific.uv.es

vinicius.rodriquesdific.uv.es

Eulogio.osetdific.uv.es

We have analyzed the $\gamma p \rightarrow p \pi^{0} \eta$ process with a particular focus on the role of a triangle singularity which appears in the decay of the $\Delta(1700)$ into $\eta \Delta(1232)$, where the $\Delta(1232)$ goes into $p$ emitting a $\pi^{0}$, while the $N(1535)$ is formed from the $\eta p$ interaction. In addition, the tree-level contribution stemming from the $\gamma p \rightarrow \Delta(1700) \rightarrow \eta \Delta(1232)$ process followed by $\Delta(1232) \rightarrow \pi^{0} p$ is taken into account. We have obtained a characteristic energy dependence from the triangle diagram in the $\pi^{0} N(1535)$ production, and a good agreement with the experimental determination up to $1.3 \mathrm{GeV}$.

XVII International Conference on Hadron Spectroscopy and Structure - Hadron 2017

25-29 September, 2017

University of Salamanca, Salamanca, Spain

\footnotetext{
* Speaker.
} 


\section{Introduction}

The $\gamma p \rightarrow \pi^{0} \eta p$ reaction is studied continuously. After a theoretical calculation [U] and early experimental measurement [[]], a theoretical study based on the dominance of $\Delta(1700)$ [B]], which is mainly generated by the $\eta \Delta$ dynamics [䧃], was found to give a good description by the following experimental study [5]. Furthermore, the analysis of the high-statistics data near the threshold is made in Ref. [6]. One interesting finding of the analysis is a rapid increase of the $\pi^{0} N(1535)$ production near the threshold though the production should be suppressed by the $p$-wave coupling.

In this study, we consider the possible role of a triangle singularity on the near-threshold behavior of the $\pi^{0} N(1535)$ production. The basic theory of the triangle singularities is given in Refs. [ $[0,[]$, and a refined formulation based on Feynman diagrams and a simple formula for the position of the triangle singularity are given in Ref. [0]. In the $\gamma p \rightarrow \pi^{0} N(1535)$ process, the triangle diagram is formed in the following way: at first the $\Delta(1700)$ decays into $\eta \Delta(1232)$ with the $\Delta(1232)$ decaying into $\pi^{0} p$ and the $\eta p$ merging into $N(1535)$. From the formula given in Ref. [Q], the diagram generates a singularity around $\sqrt{s}=1782 \mathrm{MeV}$, which corresponds to $E_{\gamma}=1220 \mathrm{MeV}$. In practice, the singularity is smeared by the width of the hadron in the intermediate state, and appears as a bump around the energy. In the following, we show the triangle singularity can explain the near-threshold energy dependence of the $\pi^{0} N(1535)$ photoproduction cross section fairly well. The details of the study are given in Ref. [ए]].

\section{Formalism and Results}

The diagrams which we consider in this study are shown in Fig. W. Figure प(a) represents

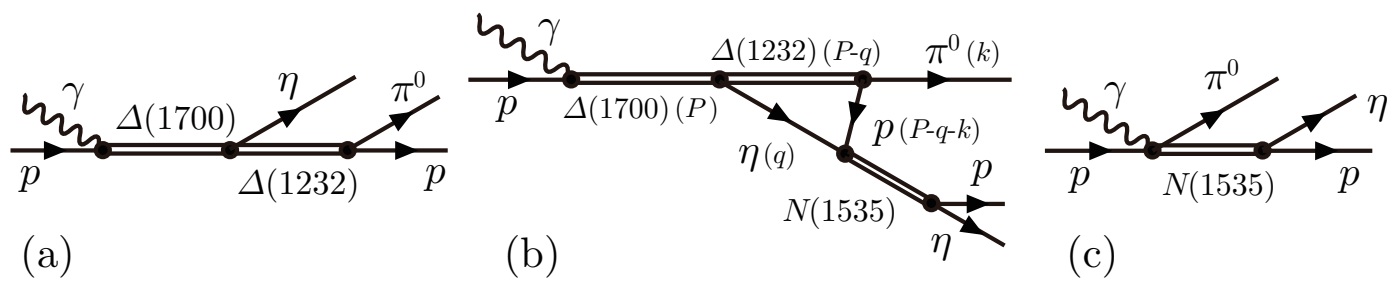

Figure 1: Diagrams for the $\gamma p \rightarrow \pi^{0} \eta p$.

the tree-level contribution which gives the major part of the $\pi^{0} \eta$ production, and Fig. $\mathbb{W}(\mathrm{b})$ is the triangle diagram on which we focus. The diagram representing the standard production mechanism which would be suppressed by the $p$-wave coupling is shown in Fig. W(c), which we will consider later for the comparison with the contribution from the triangle diagram. In Fig. W(a) and (b), the transition amplitude of $\gamma p-\Delta(1700)$ can proceed in $s$ wave. Then, the amplitude is written as $t_{\Delta^{*}, \gamma p}=g_{\Delta^{*}, \gamma p} \vec{S} \cdot \vec{\varepsilon}$ with $\vec{S}$ and $\vec{\varepsilon}$ the spin transition operator between spin $1 / 2$ and $3 / 2$ and the polarization vector of the photon, respectively. For the $\Delta-\pi^{0} p$ transition amplitude, we can write $t_{\Delta, \pi^{0} p}=\mathscr{C} f_{\pi N \Delta} / m_{\pi} \vec{S} \cdot \vec{k}$ with $\vec{k}$ the momentum of $\pi^{0}$ and $\mathscr{C}$ an appropriate Clebsch-Gordan coefficient. The coefficients of these amplitudes are fixed to reproduce the width of the $\Delta(1700)$ and $\Delta(1232)$ given in the PDG [ $[\mathrm{W}]$ ]. Regarding the mass, width, and branching fraction of the $\Delta(1700)$ to $\gamma p$, the values in the PDG [U]] have some uncertainties. Then, we fix these parameters by fitting the near-threshold data of the $\gamma p \rightarrow \pi^{0} \eta p$ cross section in the vicinity of the PDG value. 
The coupling constant of $\Delta(1700)$ to $\eta \Delta(1232)$ is taken from Ref. [田]. The amplitude is simply written as a constant, $t_{\Delta^{*}, \eta \Delta}=g_{\Delta^{*}, \eta \Delta}$, because $\eta \Delta(1232)$ and $\Delta(1700)$ can couple in $s$ wave. For the $\eta p$ elastic amplitude $t_{\eta p, \eta p}$ appearing in Fig. प(b), we use the Breit-Wigner amplitude whose parameters are taken from Ref. [[2]]. Thus, each building block of the diagram is well fixed.

The amplitudes of the diagrams in Fig. W(a) and (b) for the $\gamma p \rightarrow \pi^{0} \eta p t_{a}$ and $t_{b}$ are given as follows,

$$
\begin{aligned}
t_{a} & =g_{\Delta^{*}, \gamma p} g_{\Delta^{*}, \eta \nu} \frac{f_{\pi N \Delta}}{m_{\pi}} \sqrt{\frac{2}{3}} \frac{1}{M_{\mathrm{inv}}\left(\pi^{0} p\right)-m_{\Delta}+i \Gamma_{\Delta} / 2} \frac{1}{\sqrt{s}-m_{\Delta^{*}}+i \Gamma_{\Delta^{*}} / 2} \vec{S} \cdot \vec{k} \vec{S}^{\dagger} \cdot \vec{\varepsilon} \\
t_{b} & =2 m_{N} 2 m_{\Delta} g_{\Delta^{*}, \gamma p} g_{\Delta^{*}, \eta \Delta} t_{\eta p}, \eta p \frac{f_{\pi N \Delta}}{m_{\pi}} \sqrt{\frac{2}{3}} \frac{1}{\sqrt{s}-m_{\Delta^{*}}+i \Gamma_{\Delta^{*}} / 2} t_{T} \vec{S} \cdot \vec{k} \vec{S}^{\dagger} \cdot \vec{\varepsilon} \\
t_{T} & =\int \frac{d^{3} q}{(2 \pi)^{3}} \frac{1}{8 E_{\eta}(\vec{q}) E_{p}(\vec{q}+\vec{k}) E_{\Delta}(\vec{q})} \frac{1}{k^{0}-E_{p}(\vec{q}+\vec{k})-E_{\Delta}(\vec{q})} \frac{1}{P^{0}-E_{\Delta}(\vec{q})-E_{p}(\vec{q}+\vec{k})+i \varepsilon} \\
& \cdot \frac{2 P^{0} E_{p}(\vec{q}+\vec{k})+2 k^{0} E_{\eta}(\vec{q})-2\left[E_{\eta}(\vec{q})+E_{p}(\vec{q}+\vec{k})\right]\left[E_{\eta}(\vec{q})+E_{p}(\vec{q}+\vec{k})+E_{\Delta}(\vec{q})\right]}{\left[P^{0}-E_{\eta}(\vec{q})-E_{p}(\vec{q}+\vec{k})-k^{0}+i \varepsilon\right]\left[P^{0}+E_{\eta}(\vec{q})+E_{p}(\vec{q}+\vec{k})-k^{0}-i \varepsilon\right]}\left(2+\frac{\vec{q} \cdot \vec{k}}{\vec{k}^{2}}\right),
\end{aligned}
$$

where $E_{i}(\vec{q})=\sqrt{|\vec{q}|^{2}+m_{i}^{2}}(i=\eta, p, \Delta)$ and $P^{0}=\sqrt{s}$ is the center-of-mass energy of the initial $\gamma p$. The momentum of $\pi^{0}$ is denoted by $k$. In $t_{T}$ of Eq. (2.3]), the $q^{0}$ integral is performed analytically as in Refs. [9, [3]]. The possible form of the standard production of the $\pi^{0} N(1535)$ from $\gamma p$ in Fig. $\amalg(c) t_{c}$ would be $\vec{\varepsilon} \cdot \vec{k}$ or $(\vec{\sigma} \times \vec{k}) \cdot \vec{\varepsilon}$ which are proportional to $\vec{k}$, the $\pi^{0}$ momentum, respecting the $p$-wave coupling which comes from the conservation of the angular momentum. Averaging over the solid angle, the $\pi^{0} N(1535)$ production from the diagram Fig. W(c) has a $|\vec{k}|^{2}$ dependence.

Integrating the square of the amplitudes in Eqs. (2..J) and (2.2), with an appropriate phasespace factor, the photoproduction cross section of the $\pi^{0} \eta$ from the proton is shown in Fig. $\square$. The full result of the $\pi^{0} \eta$ production shown with the black-solid line in the figure is obtained

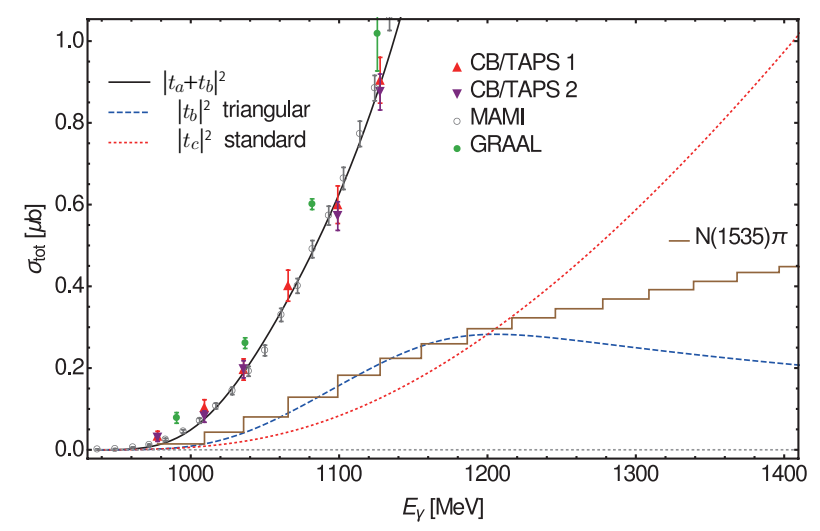

Figure 2: The $\pi^{0} \eta$ production cross section [四]. The points with error bar are the data shown in Ref. [圆], and the histogram is the partial-wave decomposition given in Ref. [目]. The magnitude of the standard production from Fig. $\mathbb{U}(\mathrm{c})$ is normalized to reproduce the $\pi^{0} N(1535)$ production at $E_{\gamma}=1200 \mathrm{MeV}$.

by tuning $m_{\Delta^{*}}, \Gamma_{\Delta^{*}}$, and $g_{\Delta^{*}, \gamma p}$, which is responsible for the branching fraction of $\Delta(1700)$ to $\gamma p$, and the experimental data for the $\pi^{0} \eta$ production are reproduced well. As one can see in the 
figure, the $\pi^{0} N(1535)$ cross section from the triangle diagram of Fig. $\mathbb{\text { (b) }}$ (shown with the bluedashed line) has a peak around $E_{\gamma}=1200 \mathrm{MeV}$, which is expected from the formula in Ref. [Q], and particularly, its low-energy behavior agrees well with the experimental determination made in Ref. [G]. Furthermore, we found a clear difference between the triangle diagram and the standard mechanism. The $\pi^{0} N(1535)$ production by the standard mechanism (shown with the red-dotted line) in Fig. $\square$ is suppressed near the threshold and increases monotonically as expected from the $|\vec{k}|^{2}$ factor.

\section{Conclusions}

In this work we have investigated the role of the triangle singularity emerging from the $\Delta(1700)$ decay into the $\eta \Delta(1232)$, with the sequential decay of the $\Delta(1232)$ to $\pi^{0} p$ and fusion of the $\eta p$ to form the $N(1535)$, which finally decays into $\eta p$. From the formula in Ref. [Q] , the triangle diagram is expected to have a singularity at $E_{\gamma}=1220 \mathrm{MeV}$, and we found a remnant of the singularity around this energy. The near-threshold behavior of the $\pi^{0} N(1535)$ production is well reproduced by the triangle diagram. In addition, we have found a characteristic energy dependence from the triangle diagram, which has a peak structure, while a suppression near the production threshold and monotonic increase are seen in the standard $\gamma p \rightarrow \pi^{0} N(1535)$ production mechanism.

\section{Acknowledgements}

S. S. acknowledges support from the program Prometeo of the Generalitat Valenciana. V.R.D. acknowledges support from the Programa Santiago Grisolia of Generalitat Valenciana (Exp. GRISOLIA/2015/005). This work is also partly supported by the Spanish Ministerio de Economia y Competitividad and European FEDER funds (Fondo Europeo de Desarrollo Regional) under the Contract Nos. FIS2014-57026-REDT, FIS2014-51948-C2-1-P, and FIS2014-51948-C2-2-P, and the Generalitat Valenciana in the program Prometeo II-2014/068.

\section{References}

[1] D. Jido, M. Oka and A. Hosaka, Prog. Theor. Phys. 106 (2001) 873.

[2] T. Nakabayashi et al., Phys. Rev. C 74 (2006) 035202.

[3] M. Doring, E. Oset and D. Strottman, Phys. Rev. C 73 (2006) 045209.

[4] S. Sarkar, E. Oset and M. J. Vicente Vacas, Nucl. Phys. A 750 (2005) 294; ibid. 780 (2006) 90.

[5] J. Ajaka et al., Phys. Rev. Lett. 100 (2008) 052003.

[6] E. Gutz et al. [CBELSA/TAPS Collaboration], Eur. Phys. J. A 50 (2014) 74.

[7] L. D. Landau, Nucl. Phys. 13 (1959) 181.

[8] S. Coleman and R. E. Norton, Nuovo Cim. 38 (1965) 438.

[9] M. Bayar, F. Aceti, F. K. Guo and E. Oset, Phys. Rev. D 94 (2016) no.7, 074039.

[10] V. R. Debastiani, S. Sakai and E. Oset, Phys. Rev. C 96 (2017) no.2, 025201.

[11] C. Patrignani et al. [Particle Data Group], Chin. Phys. C 40 (2016) no.10, 100001.

[12] T. Inoue, E. Oset and M. J. Vicente Vacas, Phys. Rev. C 65 (2002) 035204.

[13] F. Aceti, J. M. Dias and E. Oset, Eur. Phys. J. A 51 (2015) no.4, 48. 